\title{
Atmospheric-weighted temperature and its influence on cosmic ray muons
}

\author{
A Maghrabi ${ }^{1}$; M Almutayri ${ }^{1}$; H Al-Harbi ${ }^{2}$;M Baig ${ }^{3}$
}

\section{A. Maghrabi ${ }^{1}$}

National Centre for Applied Physics, King Abdulaziz City for Science and Technology, Riyadh 11442, Saudi Arabia.

E-mail: amaghrabi@kacst.edu.sa

\section{Almutayri ${ }^{1}$}

E-mail: malmutayriakacst.edu.sa

\section{H. Alharbi ${ }^{2}$}

NSRI, King Abdulaziz City for Science and Technology,

P.O.Box 6086, Riyadh 11442, Saudi Arabia.

E-mail: al harbi@kacst.edu.sa

\section{Baig ${ }^{3}$}

Physics and Astronomy Department, King Saud University,

Riyadh, Saudi Arabia,

E-mail: mbaig@ksu.edu.sa

To study the dependence of the muon rate flux on temperature we need to have some measure of atmospheric temperature above the detection site. Atmospheric-weighted temperature, known also as the effective temperature, is defined as the temperature of an atmosphere that accounts for the temperature of the real atmosphere with its varying conditions. In this paper, the influence of the atmospheric temperature on the observed muon flux was investigated and the results obtained were discussed. Cosmic ray data were obtained using a KACST muon detector, which had been in operation since 2002, located at Riyadh, Saudi Arabia (Rc is 14 GV). Radiosonde data were used to calculate two types of weighted temperature, namely dry and wet temperatures for all atmospheric profiles.

\footnotetext{
${ }^{1}$ A Maghrabi 
Atmospheric-weighted temperature and its influence on cosmic ray muons

The 34th International Cosmic Ray Conference

30 July- 6 August, 2015

The Hague, The Netherlands 


\section{Introduction}

In order to study the variations of the primary cosmic rays caused by solar and heliospheric processes, the atmospheric effects on the secondary muons observed by groundbased detectors must be considered. The rate of cosmic ray flux can be affected by two main atmospheric parameters: (1) atmospheric temperature and (2) atmospheric pressure [1-4].

Although the barometric effect correction, in which atmospheric pressure at the detector level needs to be known[5], is simple, the temperature effect is rather difficult and needs to be determined by the overall profile of the temperature at all altitudes of the atmosphere.

The effect of the atmospheric temperature on the muon count rate is usually studied by considering the temperature and/or the height of the pion production level [6-10]. On the other hand, the integrated temperature of an isothermal atmosphere with its varying conditions is sometimes calculated to study the influence of the atmospheric temperature on the detected muons. This temperature is known as the weighted or the effective temperature [11-12].

In this paper, 11 years of cosmic ray measurements collected from a KACST detector and radiosonde data were used to study the effect of atmospheric dry and wet weighted temperatures on the muon rate.

\section{Methodology}

A KACST detector is a $1 \mathrm{~m}^{2}$ plastic scintillator viewed by $120 \mathrm{~mm}$ photomultiplier tube (PMTs), which are both contained in a light-tight box. The outputs of the PMTs are amplified and digitized by a home-made A/D converter. The detector was installed at King Abdulaziz City for Science and Technology (KACST), Riyadh (lat. 24 43; long. 46 40; alt. 613 m Rc $=14$ GV), Saudi Arabia and has been in operation since 2002. More detailed descriptions of this detector are given in [13-15].Cosmic ray data during large solar flares, magnetic storms or Forbush decreases were excluded from consideration in this study.

Weighted temperatures were calculated using two definitions: dry and wet temperatures. The dry weighted temperature can be calculated using the following equation $[6,7,9]$ :

$$
T_{w d}=\frac{\int_{0}^{\infty} \frac{d(x)}{x} T(x)}{\int_{0}^{\infty} \frac{d(x)}{x}}
$$

where $\mathrm{x}$ is the atmospheric depth and $\mathrm{T}(\mathrm{x})$ is the temperature at atmospheric depths. The atmospheric depth has the unit of $\mathrm{g} / \mathrm{cm}^{2}$ and is defined as: $x=\int_{h}^{\infty} \rho(h) d(h) ; \rho(\mathrm{h})$ is the atmospheric density as a function of height (h) above the earth.

The water-vapor-weighted mean temperature of the atmosphere is approximated as [12]:

$$
T_{w w}=\frac{\int_{0}^{\infty} \frac{e_{v}}{T} d(h)}{\int_{0}^{\infty} \frac{v_{v}}{T^{2}} d(h)}
$$

where $e_{v}$ is the pressure (in $\mathrm{hPa}$ ) of water vapor calculated using the dew point temperature [16]; and $\mathrm{T}$ is the atmospheric temperature at height $\mathrm{h}$ (in Kelvin). 
The relationship between the variations of the muon intensity $(I)$ and the variations of the weighted temperature $\left(\mathrm{T}_{\mathrm{w}}\right)$ can be written as [e.g., 17]:

$$
\begin{aligned}
& \frac{\Delta I}{I_{m}}=\alpha \frac{\Delta T_{w}}{T_{w}} \\
& \frac{I-I_{m}}{I_{m}}=\alpha\left(T-T_{w m}\right)
\end{aligned}
$$

Here $\alpha$ is the temperature coefficient and $I_{m}$ (159.31 in this study) and $T_{w m}$ are the mean values of the muon rate and the weighted temperature during the study period, respectively.

\section{Results}

Routine radiosonde data from Riyadh airport for the period of study were used to calculate the atmospheric dry and wet weighted temperature overall profile of the atmosphere using equations 1 and 3, respectively. Corresponding pressure-corrected muon measurements were used. The barometric coefficient used for correcting the data for pressure was $0.18 \% / \mathrm{hPa}$ in accordance with our previous extensive study [5].

Figure 1 shows the inter-annual variations of the measured muon rate versus the (a) dry weighted temperature $\mathrm{T}_{w d}$ and (b) wet weighted temperature $\mathrm{T}_{w w}$, from July 2002 to March 2012. The $\mathrm{T}_{w d}$ ranges between $225 \mathrm{~K}$ and $235 \mathrm{~K}$ with a mean value of $230.12 \pm 2.1 \mathrm{~K}$, whereas $\mathrm{T}_{w w}$ is confined between 273 and 298 with a mean of $287.52 \pm 6.2 \mathrm{~K}$. It is noticeable that the $\mathrm{T}_{w w}$ are higher than the $\mathrm{T}_{w d}$ by about 58 degrees and have higher experimental error. Although the two temperatures were calculated using different methods, they are both anticorrelated with the measured cosmic ray muons. While the strength of this relation between the two parameters is different from one year to another, the association is obvious throughout all years. These results are consistent with those previously found for different experiments [4,7-8].

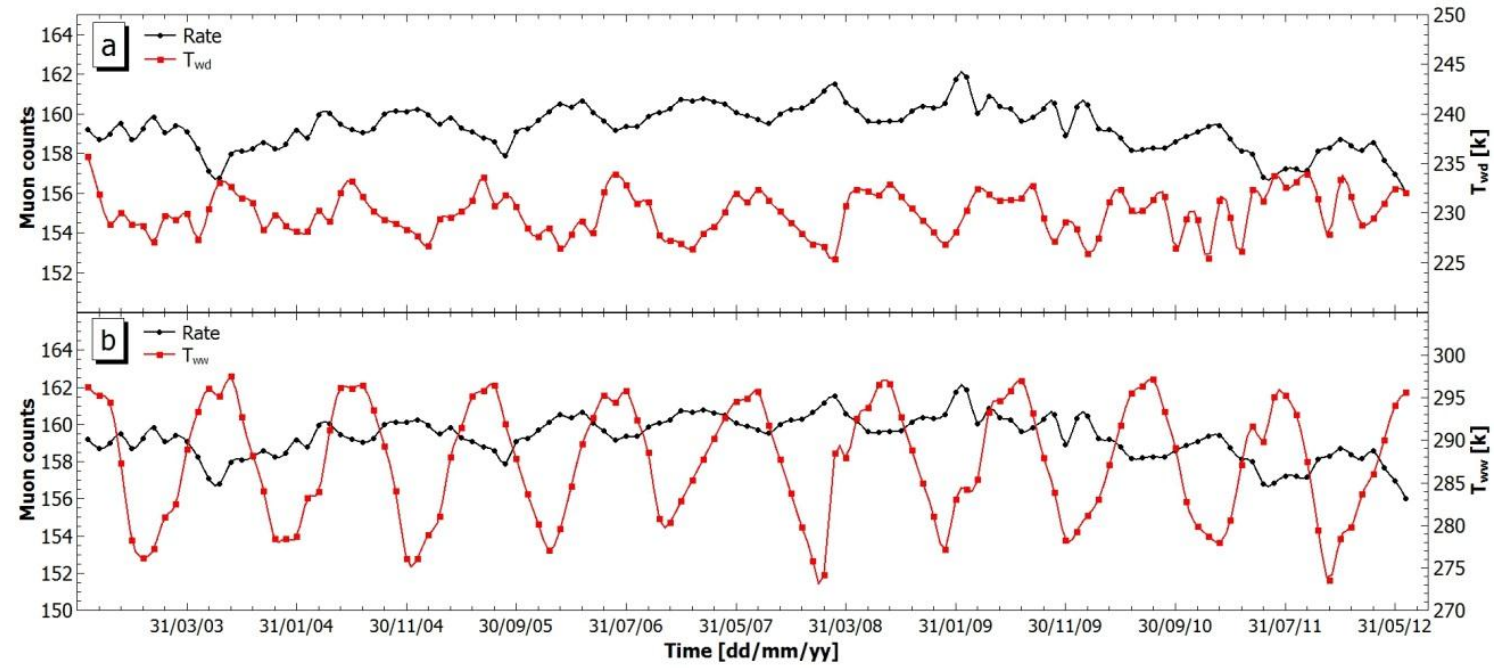

Figure 1: One month average counting rate of cosmic ray muons versus (a) Twd and (b) Tww for the period between 2002 and 2012.

Figure (2) is a scatter plot between the $\frac{\Delta I}{I_{m}}$ against (a) $\frac{\Delta T_{w d}}{T_{w d}}$ and (b) $\frac{\Delta T_{w w}}{T_{w w}}$. The straight line is the line of best fit between the two parameters. Although there is spread in the data, the negative relationship between the two temperatures and the muon rates is apparent. Regression analysis between $\frac{\Delta I}{I_{m}}$ and $\frac{\Delta T_{w d}}{T_{w d}}$ for the whole data gives a value of $\alpha_{w d}=-0.14 \pm 0.002[\% / \mathrm{K}]$ with 
a correlation coefficient of 0.52 . On the other hand, $\alpha_{w w}$ has a value of $-0.031 \pm 0.001[\% / \mathrm{K}]$ and a correlation coefficient of only 0.31 .

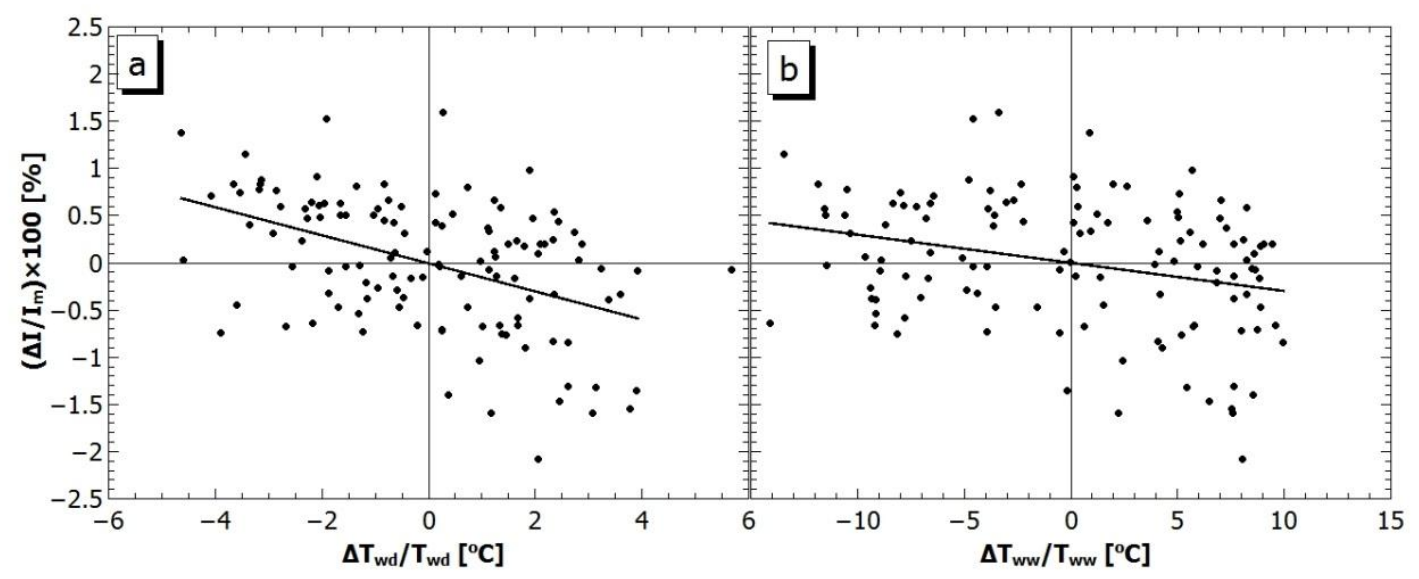

Figure 2: Regression results between one month mean muon variations $\Delta I / I_{m}$ versus (a) $\Delta T_{w d} / T_{w d}$ and (b) $\Delta T_{w w} / T_{w w}$ for the period between 2002 and 2012 .

Figure (3) shows the monthly variations of the measured muon rate versus the (a) $\mathrm{T}_{w d}$ and (b) $\mathrm{T}_{w w}$. The seasonal variations between the muon rates and both temperatures are clearly observed. The muon rate decreases as the temperatures increase. The negative temperature effect on the cosmic ray muons is due to the expansion of the atmosphere as a result of the atmospheric temperature increasing during summer times causing longer distances to be travelled by the decayed muons and the opposite occurring during winter times. This relationship between the two parameters is presented in Figure 4. Regression results give values of $\alpha_{w d}=-0.176 \pm 0.02[\% / / \mathrm{K}]$ and $-\alpha_{w w}=0.038 \pm 0.005[\% / / \mathrm{K}]$ with correlation coefficients of 0.94 and 0.86 , respectively. Although using the monthly data gives better correlation coefficients than the inter-annual data, the temperature coefficients obtained from both data sets do not differ much from each other. To compare our results with some other studies, muon data obtained from URGAN hodoscope [7] estimated a value of $\alpha_{w d} \approx-0.23 \%$ [K]; and [1] presented a value of $0.26 \%[\mathrm{~K}]$. On the other hand, underground muon detectors presented positive temperature coefficients $\alpha_{w d}$ [17-18]. No previous works have been reported to calculate the water-vapor-weighted temperature. 

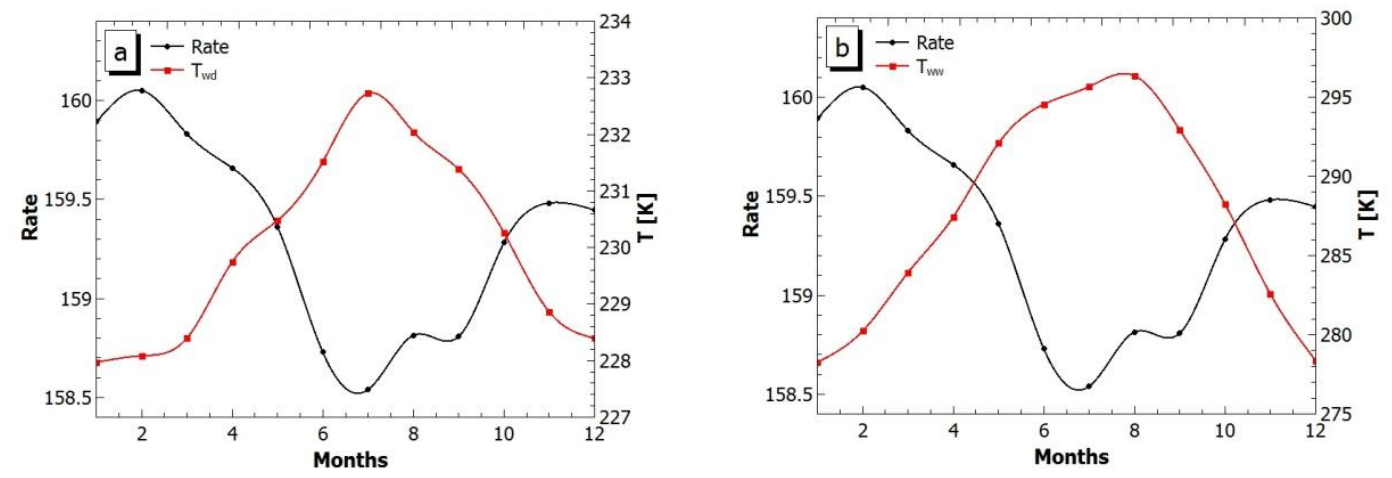

Figure 3: Superposition of the monthly mean of the cosmic ray muon rates versus (a) $T_{w d}$ and $(b) T_{w d}$, over the period 2002-2012.

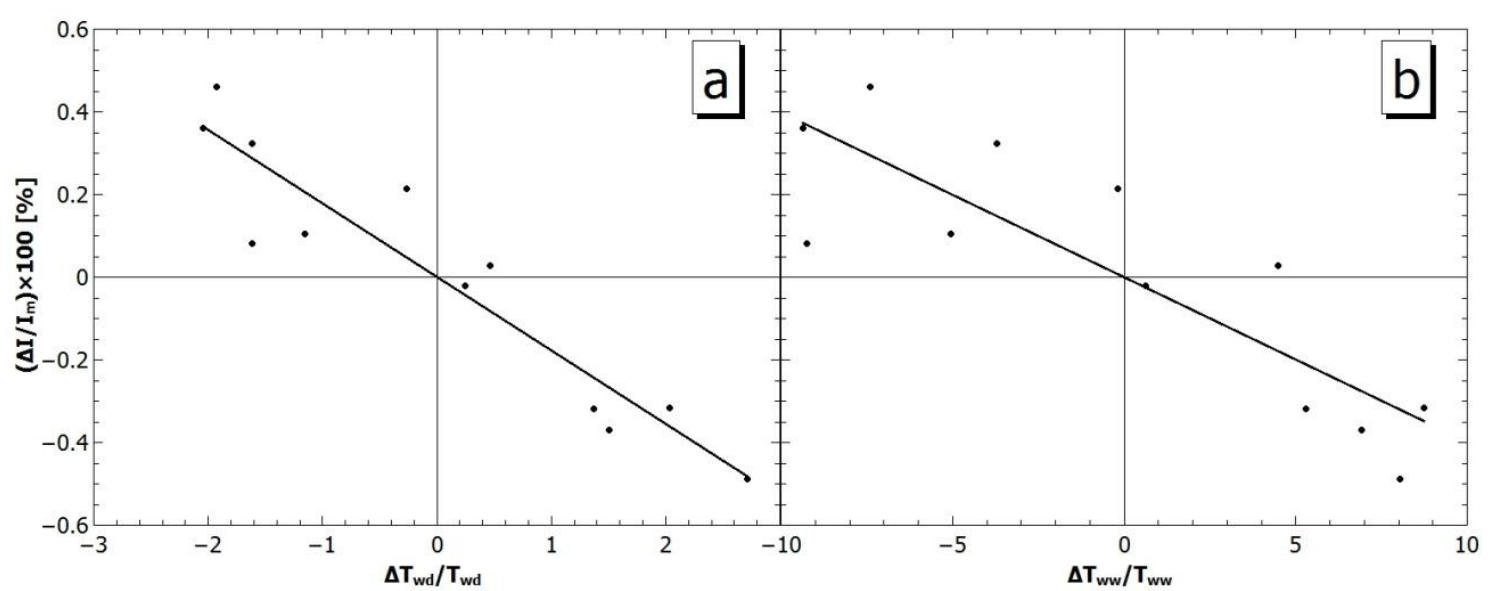

Figure 4: Same as Figure (2) but for the monthly mean values (data of Figure 3) of the muon variations $\Delta I / I_{m}$ versus (a) $\Delta T_{w d} / T_{w d}$ and (b) $\Delta T_{w w} / T_{w w}$ for the period between 2002 and 2012.

Figure 5 shows the corrected muon counts over a period of two years using the two weighted temperature coefficients. We can see that the correction for the temperature effect does not exceed more than $1 \%$. Additionally, we can see that the correction for the temperature effect did not remove the seasonal variations of the detected muon and other causes of this seasonality need to be investigated. 


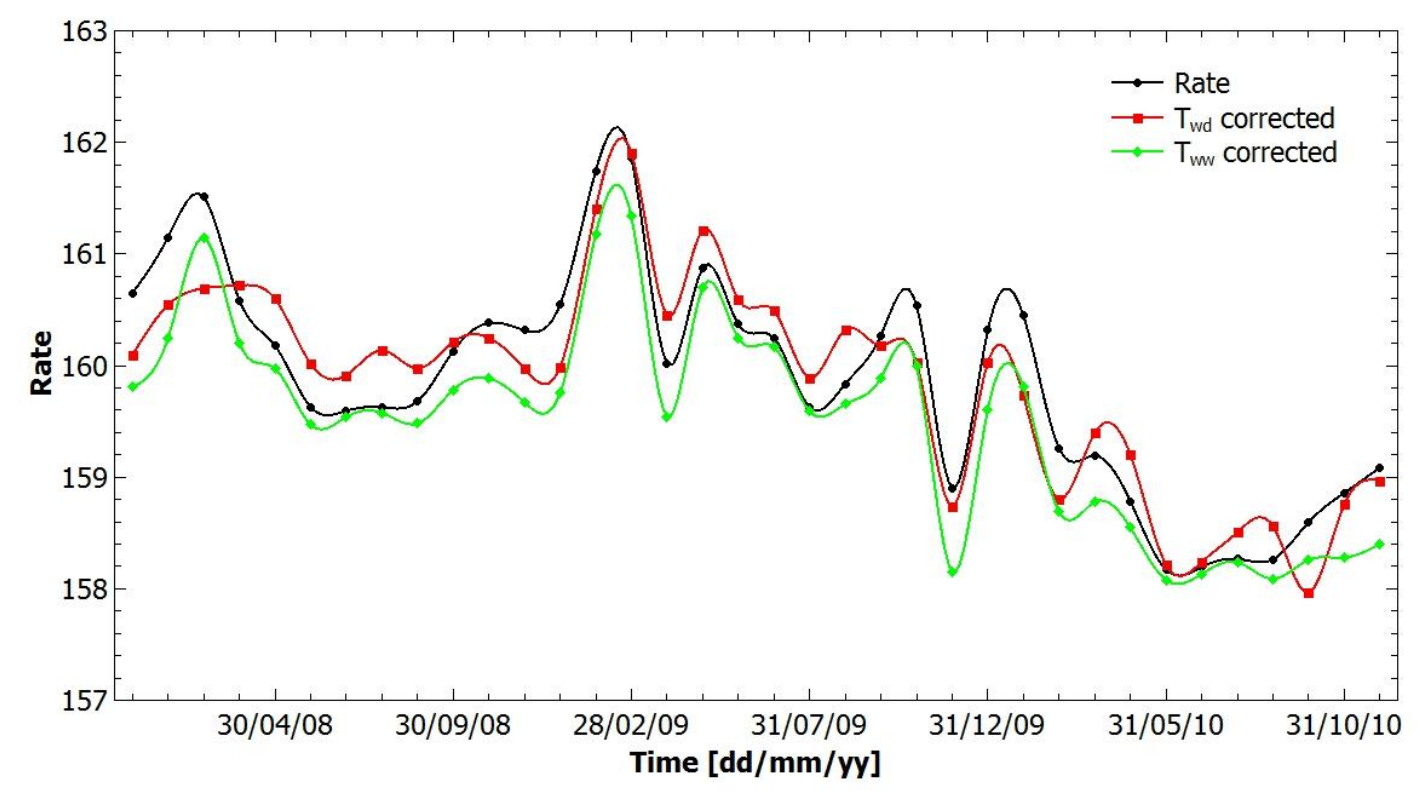

Figure 5: Muon rate compared with the rate corrected using $T_{w d}$ and $T_{w w}$.

\section{Conclusions:}

This paper provides our next step in the atmospheric correction of cosmic ray muons, namely correction due to integrated atmospheric temperatures. Cosmic ray measurements from 2002 to 2012 were obtained from a KACST muon detector. Corresponding radiosonde data were used to calculate the water vapor and dry atmospheric-weighted temperatures. Although the former temperatures were warmer than the latter, it was found that the cosmic ray muon rate is anticorrelated with both temperatures. This relationship was much clearer in the monthly data than the inter-annual scale. Our results are comparable with those obtained previously by different investigators.

\section{Acknowledgments}

The authors would like to thank King Abdulaziz City for Science and Technology (KACST) for supporting this work.

\section{References}

[1] DeMendonça et al., Analysis of atmospheric pressure and temperature effects on cosmic ray measurements, J. Geophys. Res 118, 1403. 2013

[2] Duperier, A. The meson intensity at the surface of the Earth and the temperature at the production level, Proc. Phys. Soc., 62A, 684-696, 1949. 
[3] Maeda, K., and M. Wada, Atmospheric temperature effect upon cosmic ray intensity at sea level, J. Sci. Res. Ins. (Tokyo), 48, 71-79, 1954.

[4] Yanke, V. et al., (2011), Temperature effect of general component seen by cosmic ray detectors, Proceedings of 32nd International Cosmic Ray Conference, 11, 377-380.

[5] A. H. Maghrabi, et al., Influence of the Atmospheric Mass on the High Energy Cosmic Ray Muons during a Solar Cycle, Advances in Astronomy doi.org/10.1155/2015/939146

[6] A Maghrabi; et al., The Ground Temperature Effect on Cosmic-Ray Muons at Mid latitude City, Proc.23d ECRS ,2014.

[7] A N Dmitrieva, et al., Temperature effect correction for muon flux at the Earth surface: estimation of the accuracy of different methods, 23rd ECRS and 32nd Russian Cosmic Ray Conference ; doi:10.1088/1742-6596/409/1/01213

[8] C R Braga et al; Temperature effect correction for the cosmic ray muon data observed at the Brazilian Southern Space Observatory in São Martinho da Serra, 23rd ECRS and 32nd Russian Cosmic Ray Conference doi:10.1088/1742-6596/409/1/012138

[9] Baker, C.P., et al., Atmospheric correction analysis for the Mawson muon telescopes, Proc. of 23th ICRC, Calgary, 3, p. 753, 1993

[10] Dvornikov, V.M., et al., Determination of the mass-average temperature on the cosmic ray intensity data, Geomag. Aeron., 16 (5), 923-925, 1976.

[11] Bevis, M., S. et al., , GPS meteorology: Remote sensing of atmospheric watervapor using the Global Positioning System, J. Geophys. Res., 97(D14), 15,787 - 15,801,1992.

[12] Wang J, et al., Global estimates of water-vapor-weighted mean temperature of the atmosphere for GPS applications, J.G.R. 110, D21101, doi:10.1029/2005JD006215,2005.

[13] Alghamdi , A ; et al.,, Designing and constructing of a two scintillator crystal rotatable telescope for muon flux variation studies, Proc. SPIE 9154, doi:10.1117/12.2059232; 2014.

[14] Maghrabi A, et al., The KACST muon detector and its application to cosmic-ray variations studies; Adv. Space Res.50 ,700. 2012,

[15] Maghrabi A, et al., On the calibration of a single channel cosmic ray particle detector, Proc. SPIE 9154, 91542K, 2014

[16] Maghrabi A. H, and Al Dajani, H.M, Estimation of precipitable water vapour using vapour pressure and air temperature in an arid region in central Saudi Arabia, Journal of the Association of Arab Universities for Basic and Applied Sciences 14, 1-8;2013

[17] Ganeva, M., et al., Seasonal variations of the muon flux seen by muon telescope MuSTAnG. 23rd ECRS and 32nd Russian Cosmic Ray Conference

[18] Ambrosio, M., et al. , Seasonal variations in the underground muon intensity as seen by MACRO, Astroparticle Phys., 7(1-2), 109-124, 1997. 